\title{
Nanoscale
}

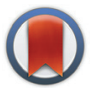

CrossMark

\&lick for updates

Cite this: Nanoscale, 2015, 7, 2281

Received 28th October 2014,

Accepted 2nd January 2015

DOI: $10.1039 / c 4 n r 06356 b$

www.rsc.org/nanoscale

\section{Molecular interfaces for plasmonic hot electron photovoltaics $\uparrow$}

\author{
F. Pelayo García de Arquer, Agustín Mihiț and Gerasimos Konstantatos*
}

The use of self-assembled monolayers (SAMs) to improve and tailor the photovoltaic performance of plasmonic hot-electron Schottky solar cells is presented. SAMs allow the simultaneous control of open-circuit voltage, hot-electron injection and shortcircuit current. To that end, a plurality of molecule structural parameters can be adjusted: SAM molecule's length can be adjusted to control plasmonic hot electron injection. Modifying SAMs dipole moment allows for a precise tuning of the open-circuit voltage. The functionalization of the SAM can also be selected to modify short-circuit current. This allows the simultaneous achievement of high open-circuit voltages $(0.56 \mathrm{~V})$ and fill-factors $(0.58)$, IPCE above $5 \%$ at the plasmon resonance and maximum power-conversion efficiencies of $0.11 \%$, record for this class of devices.

\section{Introduction}

The unique light-matter interaction of plasmonic systems has seen an increasing interest in the field during the last decades, with applications in bio-sensing, ${ }^{1}$ photodetection, ${ }^{2}$ and light energy harvesting. ${ }^{3}$ In particular, the use of metallic nanostructures has proved beneficial in a number of systems, from photovoltaics $^{4-6}$ to photodetectors. ${ }^{2,7,8}$ The enhanced performance in these devices typically stems from the superior light trapping characteristics of such structures, which yield increased absorption in the surrounding semiconductors. In those approaches, metal nanostructures act as passive elements that introduce parasitic ohmic losses. However, it was shown recently that, a direct photoelectric energy conversion from light absorbed in the metal is within reach, by properly harnessing the hot-electron population derived from the Landau damping of these plasmonic resonances, ${ }^{9-20}$ This

ICFO - Institut de Ciències Fotòniques, Mediterranean Technology Park 08860 Castelldefels, Barcelona, Spain.E-mail: gerasimos.konstantatos@icfo.es

$\dagger$ Electronic supplementary information (ESI) available: Contact-potential differentiometry measurements, FTIR characterization, performance statistics and gold devices. See DOI: 10.1039/c4nr06356b

\$Present Address: Department of Enginyeria Electrònica, Elèctrica i Automàtica, Universitat Rovira i Virgili, 43007 Tarragona, Spain. opens the exciting possibility of a new sensing and light harvesting technology, whose spectral response can be tailored by properly modifying the topology of a metal nanostructure, and is beyond the band-to-band absorption paradigm in traditional semiconductors. ${ }^{11}$ Theoretical predictions set maximum photovoltaic power conversion efficiencies that range from $10 \%$ to $22 \%$ depending on the applied model for hot electron population and emission. ${ }^{21,22}$ Reported experimental values are, however, far from this limit challenging the community for new advances in plasmonic hot-electron photovoltaics.

One critical step in order to achieve efficient hot-electron optoelectronic devices is the collection of the plasmonically derived hot-electron population before it thermalizes via the coexisting electron-electron and electron-phonon scattering. ${ }^{18,23-25}$ To this end, metal-semiconductor (MS) Schottky junctions have been employed, ${ }^{11,12,26}$ that take advantage of the built-in field in the vicinity of the metal nanostructure to separate the photogenerated carriers. The use of metalinsulator-metal architectures was also successfully employed for hot-electron photodetection. ${ }^{15,16}$ Photovoltaic devices require the concurrent achievement of an open-circuit voltage $\left(V_{\mathrm{oc}}\right)$ and a short-circuit current $\left(J_{\mathrm{sc}}\right)$, which was hindered in previous works by detrimental interface states resulting a Fermi-level pinning and the suppression of $V_{\text {oc }}$. The crucial role of the metal-semiconductor interface for photoelectric energy conversion and photovoltaics was recently reported, ${ }^{9}$ where it was shown that, by the inclusion of an ultrathin $\mathrm{Al}_{2} \mathrm{O}_{3}$ insulator layer, a high $V_{\text {oc }}$ and $\mathrm{FF}$ can be achieved. This inorganic based approach, however, limits the roadmap of plasmonic hot-electron solar cells for it does not allow the simultaneous control over $V_{\mathrm{oc}}$ and $J_{\text {sc }}$, an important step required in exploiting the exotic physics of these devices for reaching higher performance. Instead, a more versatile approach can be envisaged through the exploitation of molecular species in self assembled monolayers. ${ }^{27}$ Molecules, unlike inorganic wide bandgap semiconductors, possess a plurality of structural parameters that can be tuned in these systems: the length of the molecule, the conjugated or aliphatic character and the end-functional groups that serve to 
strongly bind on a given surface and passivate electronic defect states. ${ }^{27-35}$ Not to exclude also their solution processability which is a significantly lower cost manufacturing process compared to atomic layer deposition.

In this work we exploit these unique properties of SAM of molecular species to tune the electronic properties of the molecular interface in a plasmonic hot-electron photovoltaic device to simultaneously control $J_{\mathrm{sc}}$ and $V_{\mathrm{oc}}$. We demonstrate that hot-electron injection efficiency, and thus $J_{\text {sc }}$, can be modified in a twofold manner by either adjusting the molecular length or their head-functionalization and that a highly precise degree of control can be exerted over the open-circuit voltage by modifying the interface dipoles formed by the SAM. By doing so we report a PCE $=0.11 \%$, the highest up to date to the best of our knowledge for plasmonic-hot electron photovoltaic devices, with high open-circuit voltages and incident photon conversion efficiencies (IPCE) in excess of $5 \%$ at the plasmon resonance peak.

\section{SAMs to passivate the metal-semiconductor interface}

The solar cell structure is shown in Fig. 1a. Briefly, a titanium dioxide nano-crystalline film electron transport layer is deposited on top of a transparent conductive oxide. The cross sectional scanning electron micrograph (SEM) reveals that the $\mathrm{Ag}$ follows $\mathrm{TiO}_{2}$ roughness, in the order of 10-80 nm (ref. 9), and enables the coupling of incident light to $\mathrm{Ag}$ plasmonic resonances. The optical excitation of the plasmonic resonance eventually results in a hot-electron population within the metal. $^{37}$

For untreated $\mathrm{TiO}_{2}$ films, the presence of surface states due to the existence of dangling bonds and off-stoichiometry defects results in a charge localization that modifies the surface potential and prevents the formation of a built-in potential in the eventual contact with the metal electrode (Fig. 1b). ${ }^{9}$ However, these defects can be overcome by passivating the $\mathrm{TiO}_{2}$ surface with a set of molecules that containing a compatible functional group give rise to a SAM over its surface
(Fig. 1c). ${ }^{30,38,39}$ In our case we employ carboxylic acid (R-COOH) functionalized molecules, a commonly used binding group to $\mathrm{TiO}_{2}$. Once grafted to the semiconductor surface, the passivation of midgap states is observed as the Fermi level is shifted (see ESI S1 $\dagger$ ).

The adsorption of an oleic acid $(\mathrm{OA})\left(\mathrm{C}_{18} \mathrm{H}_{36} \mathrm{O}_{2}\right)$ SAM to the $\mathrm{TiO}_{2}$ has been monitored with Fourier Transform Infrared spectroscopy (FTIR) by looking at the different vibrational characteristics of the molecules (see Fig. 2). The difference in the symmetric and asymmetric stretching frequencies of the deprotonated carboxyl group (of the order of $100 \mathrm{~cm}^{-1}$ ) suggests a bidendate chelating binding mode of $\mathrm{COOH}$ to $\mathrm{TiO}_{2}{ }^{36}$ In this way each $\mathrm{C}_{18} \mathrm{H}_{36} \mathrm{O}_{2}$ molecule will bind to a $\mathrm{Ti}$ atom in the $\mathrm{TiO}_{2}$ slab. After substrate functionalization the

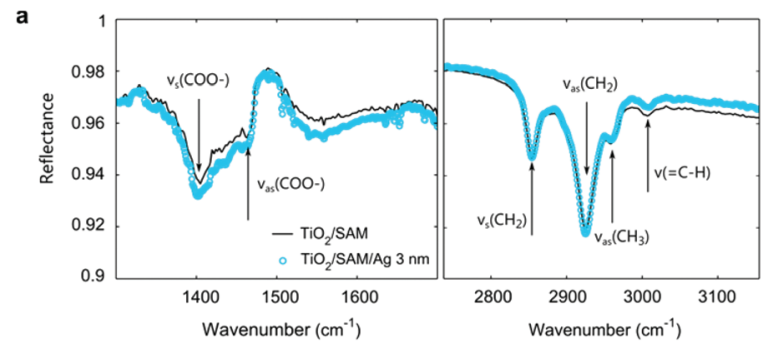

b $\mathrm{TiO}_{2}-\mathrm{COOH}$ binding modes
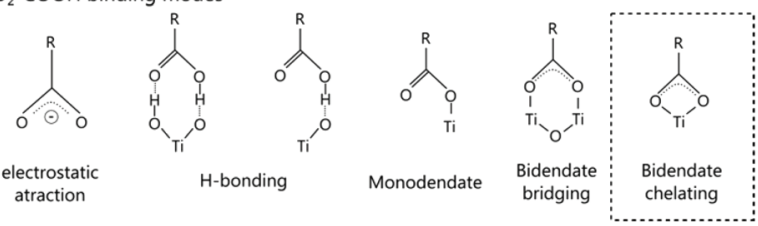

Fig. 2 Molecular bridging mode and integrity of SAM after metal deposition. (a) FTIR reflectance spectra of a TiO2/SAM sample before (solid line) and after (circle marked dots) the deposition of an ultrathin Ag layer $(3 \mathrm{~nm})$. (b) Possible molecule binding modes. The difference in the symmetric and asymmetric stretching frequencies of the deprotonated carboxyl group of the order of $100 \mathrm{~cm}^{-1}$ suggest a bidendate chelating binding mode of $\mathrm{COOH}$ to $\mathrm{TiO}_{2}{ }^{36}$ In this way each $\mathrm{C}_{18} \mathrm{H}_{36} \mathrm{O}_{2}$ molecule will bind to a $\mathrm{Ti}$ atom in the $\mathrm{TiO}_{2}$.
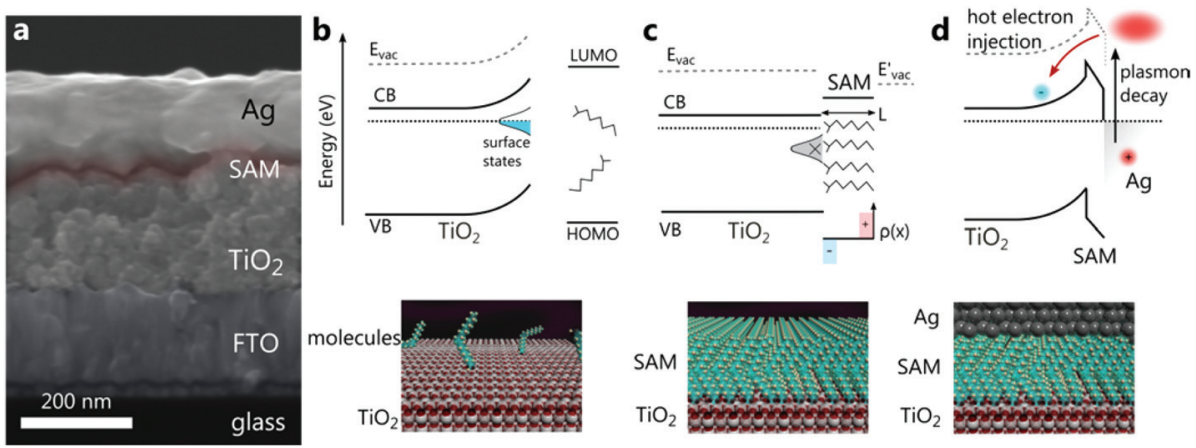

Fig. 1 Molecular interfaces for plasmonic hot electron photovoltaics. (a) Cross-sectional SEM of a plasmonic hot-electron solar cell, comprising a transparent conductive bottom electrode, a high bandgap semiconductor layer $\left(\mathrm{TiO}_{2}\right)$ which is covered by a molecular self-assembled monolayer (SAM). An Ag electrode is used to harvest the hot-electrons generated by plasmonic damping. Without the presence of the $\mathrm{SAM}^{(b)} \mathrm{TiO}_{2}$ defect states create a space charge region which would result in Fermi level pinning and no photovoltaic effect upon contact. (c) The SAM allows the passivation of these detrimental states and the simultaneous control over the interface conformation and charge distribution. (d) This ultimately allows the control over the photovoltaic figures of merit of the plasmonic hot-electron devices. 
most critical step in the fabrication of SAMs junctions is the deposition of the top electrode, as it can compromise the integrity of the underlying molecular layer. The influence of the deposition conditions has been widely studied in other systems, ${ }^{28,40-43}$ and soft-electrode transfer or indirect evaporation reported as the less harming methods to the SAM. In our case we have also found that direct metal evaporation is capable of maintaining the quality SAMs for low enough deposition rates and temperatures (see Experimental section for more details). In order to assess the integrity of the OA-metal buried interface, we first cross-compared the vibrational modes of the SAM in a $\mathrm{TiO}_{2}$-SAM configuration before and after metal deposition. We started by monitoring how the SAM is affected during the first, more aggressive nanometers, where the bare SAM is exposed to the impinging metal atoms. To do that, we deposited $3 \mathrm{~nm}$ of $\mathrm{Ag}$ on top of the SAM and measured by FTIR in an attenuated total reflection configuration the absorption of the SAM. ${ }^{44}$ The coincidence of the characteristic stretching frequencies for COO-, $\mathrm{CH}_{2}$ and $\mathrm{CH}_{3}$ suggest that the SAM has not been substantially modified after the first steps of electrode deposition (Fig. 2). The bridging mode of the SAM constituent molecules remains bidendate chelating (Fig. 2b) and both the $\mathrm{CH}_{2}$ backbone and the $\mathrm{CH}_{3}$ molecule end are not damaged. To further characterize the quality of the SAM after the full deposition of the final electrode (where FTIR cannot be employed since both front and back electrodes are opaque in the relevant IR frequencies), we sought to take advantage of the strong fields associated with the plasmonic resonances in the $\mathrm{Ag}$ textured electrode, and measured the SAM vibrational modes by plasmon-enhanced Raman spectroscopy from the FTO side (Fig. 3a). In doing so we found that both the $\left(\mathrm{CH}_{3}\right)$ and $\left(\mathrm{CH}_{2}\right)$ stretching frequencies were coincident in both cases, which pointed towards a majorly undamaged SAM in the final configuration. The photovoltaic performance of a representative OA device is shown in Fig. $3 \mathrm{~b}$, yielding a $V_{\text {oc }}$ of $0.36 \mathrm{~V}$. The average power conversion efficiency (PCE) of these devices is $0.05 \%$. The superior performance of this approach, compared to inorganic passivation schemes, ${ }^{9}$ stems from the higher $J_{\mathrm{sc}}(0.264$ versus $0.140 \mathrm{~mA} \mathrm{~cm}{ }^{-2}$ ) of these devices and points towards a more efficient hot-electron injection from the plasmonic electrode. The IPCE is shown in Fig. 3c. The spectral response is clearly dominated by the plasmonic character of the textured electrode and shows a maximum value of $2.1 \%$ at resonance. Flat unpatterned control references consisting of OA functionalized $\mathrm{TiO}_{2}$ atomically-layer-deposited substrates show on the other hand no significant contribution in the visible region of the spectrum. We note that in the absence of OA no measurable photovoltaic response was monitored. ${ }^{9}$

\section{SAMs to control hot-electron injection and $J_{\text {sc }}$}

Once the ability of the SAMs to correct detrimental interfacial states-shown to be critical for the photovoltaic performance of $\mathrm{TiO}_{2}$ metal plasmonic solar cells-has been demonstrated, we proceed now to study how both the electronic properties and hot-electron injection mechanism can be controlled in
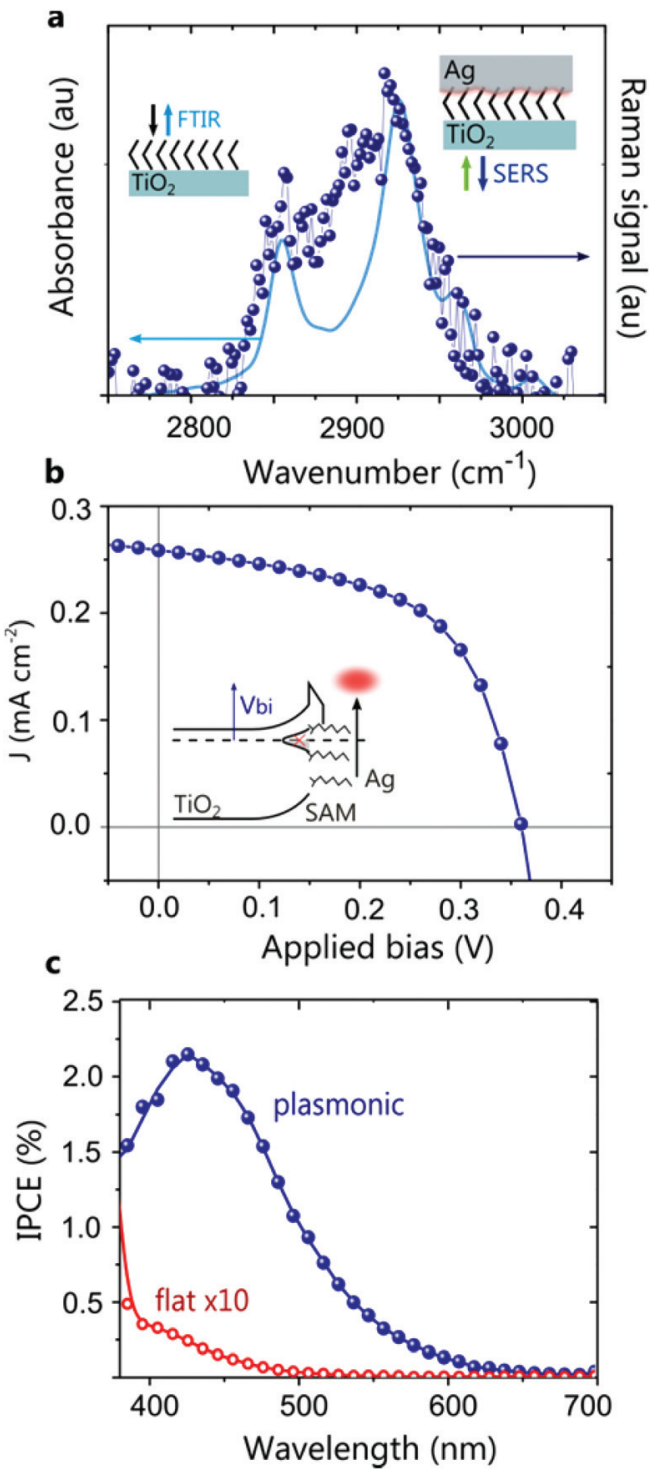

Fig. 3 Molecular interfaces to passivate interfacial states. (a) Vibrational signatures of the grafted oleic acid $\left(\mathrm{C}_{18} \mathrm{H}_{36} \mathrm{O}_{2}\right)$ self-assembled monolayer (SAM) before and after metal electrode deposition. (b) Currentvoltage characteristics under simulated solar illumination AM 1.5 G showcasing the photovoltaic performance of these devices due to midgap states passivation. (c) The IPCE of these devices illustrates that the dominant photocurrent generation mechanism is the injection of plasmonic hot electrons from the Ag metal electrode.

these devices by the modification of the SAM. For that purpose we started by selecting a set of carboxyl aliphatic chains of different lengths of the form of $\mathrm{R}-\mathrm{COOH}$, where the number of carbons and saturation of the $\mathrm{R}$ chain is modified (Fig. 4a). In this way, the $\mathrm{COOH}$ functional group will bind to the $\mathrm{TiO}_{2}$ surface as shown in the OA case, exposing the unfunctionalized methyl end to the $\mathrm{Ag}$ metal electrode.

The adhesion of the different SAMs to the $\mathrm{TiO}_{2}$ was verified by FTIR for all molecules (see ESI S2†). The thickness of the resulting SAM will modify charge transport and injection across the nanoscopic junctions, thus offering the possibility 
a

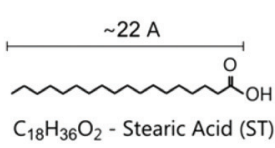

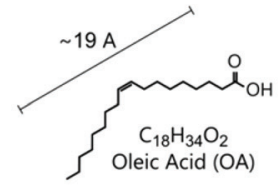

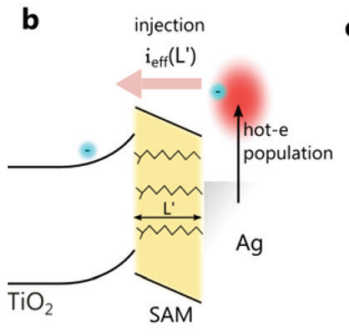

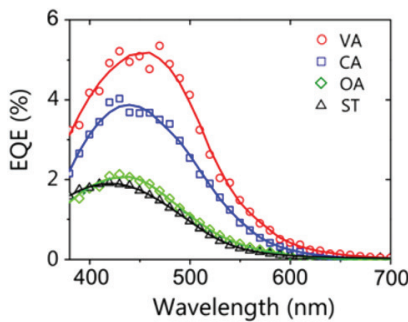

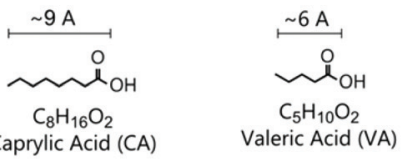

d

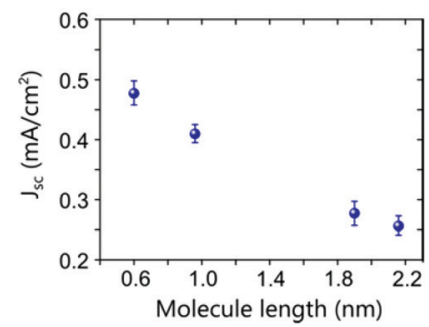

Fig. 4 Molecular length to control hot-electron injection efficiency. (a) The length of the SAM constituent molecules will determine its thickness, and can therefore be controlled by selecting a proper set of molecules. As a consequence, the hot-electron injection efficiency (b) is expected to change as the $\mathrm{Ag}-\mathrm{TiO}_{2}$ distance diminishes. The IPCE increases as the length of the SAM decreases, from $18 \mathrm{C}$ (stearic acid) to $5 \mathrm{C}$ (valeric acid) yielding a maximum value beyond $5 \%$. (c). This is correlated with the increase in short-circuit current (d), from 0.25 up to $0.475 \mathrm{~mA} \mathrm{~cm}^{-2}$.

to modulate hot-electron injection efficiency $\left(i_{\text {eff }}\right)$ (Fig. $\left.4 \mathrm{~b}\right)$. The IPCE for the different molecules is shown in Fig. 4c, demonstrating the correlation of the injection efficiency with the length of the molecules. Longest molecules (such as stearic acid, with 18 carbons and an approximate length of $22 \AA$ ) yield the lowest IPCEs, around $2 \%$, whilst the maximum IPCE (in excess of 5\%) is reached with the shortest length molecule (valeric acid (VA), 5 carbons and approximately $6 \AA$ ). The same trend is observed for the short-circuit current (Fig. 4d), which increases from $0.26 \mathrm{~mA} \mathrm{~cm}^{-2}$ to $0.44 \mathrm{~mA} \mathrm{~cm}^{-2}$ (average values). A maximum PCE of $0.10 \%$ and $J_{\mathrm{sc}}=0.50 \mathrm{~mA}$ $\mathrm{cm}^{-2}$ are obtained for VA. The relevant figures of merit for this set of devices are shown in Table S3. $\dagger$ The IPCE for an Au electrode with the same SAM is also shown in Fig. S4, $\uparrow$ showcasing the different contribution from the $\mathrm{Au}$ plasmonic resonance and an over an order of magnitude improvement compared to the best inorganic interfaces. ${ }^{9}$ It can be seen, interestingly, that there is also a dependence of the open-circuit voltage with molecules length. Due to the similar dipole moment of the molecules under study, this trend could be accounted for by a different packing, orientation and/or density of the molecules within the SAM. As the textured nature of our substrates impedes a proper characterization of the SAM density, we consider now a set of molecules with similar geometries but different dipole moments.

\section{SAMs to concurrently tailor open-circuit voltage and hot-electron injection}

To get further insights into the ability of the SAM to modify the open-circuit voltage in hot-electron solar cells, we proceed now to study a set of molecules with fixed structural parameters but different chemical functionalities (HOOC- $\mathrm{C}_{6} \mathrm{H}_{4}-\mathrm{X}$ ), such that their dipole moment can be tuned independent of their length, by the selection of different functional species in both molecular ends. The electrostatic potential induced by the molecule's dipole will result in a local modification of the charges across the interfaces and the vacuum level, thus enabling the nanoscale control over the band alignment and electrostatic fields along the interface (see Fig. 5a). In this way

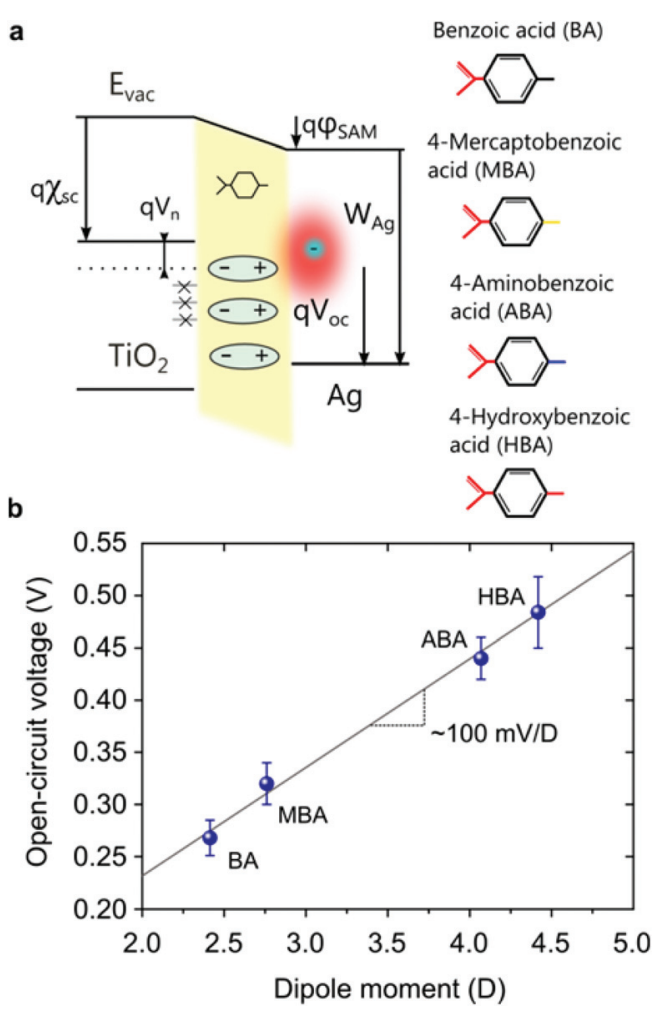

Fig. 5 Molecular dipoles to control open-circuit voltage. (a) The electrostatic potential of the SAM can modify the local vacuum level also changing the effective barrier after contact. The resulting opencircuit voltage can therefore be tuned by selecting molecules of different dipole moments. (b) There is a clear correlation of the $V_{\text {oc }}$ with molecule's dipole, which enables to linearly control the $V_{\text {oc }}$ with a rate of $104 \mathrm{mV} \mathrm{D}^{-1}$. 
we can expect the barrier and the injection to be dominated by the dipole moment as described in eqn (1).

$$
\mathrm{q} V_{\mathrm{oc}}=W_{\mathrm{Ag}}-\left(\chi_{\mathrm{sc}}+\mathrm{q} V_{\mathrm{n}}-\mathrm{q} \phi_{\mathrm{SAM}}\right)
$$

where $W_{\mathrm{Ag}}$ is the metal work function, $\chi_{\mathrm{sc}}$ is the semiconductor electron affinity, $\mathrm{q} V_{\mathrm{n}}$ is the energy difference from the Fermi to the semiconductor conduction band and $\mathrm{q} \phi_{\mathrm{SAM}}$ is the contribution from the SAM. The latter can be further written as,

$$
\Phi_{\mathrm{SAM}}=\frac{N \mu \cos (\theta)}{\varepsilon_{0} \varepsilon_{\mathrm{r}}}
$$

and depends on the surface density of dipoles $(N)$, their magnitude $(\mu)$ and orientation angle $(\theta)$. Therefore the open-circuit voltage can be increased by selecting a set of molecules such that once attached to the $\mathrm{TiO}_{2}$ lower the local vacuum level in the other end (i.e. a negative dipole moment) resulting in an increase of the net barrier after contact. ${ }^{45,46}$ The results are shown in Fig. 5b. Conjugated molecules with lower dipole moment (benzoic acid and 4-mercaptobenzoic acid) yield consistently the lower open-circuit voltages $(0.26 \mathrm{~V}$ and $0.32 \mathrm{~V}$ respectively). Molecules with higher dipole moments, such as 4-aminobenzoic (ABA) acid and 4-hydroxybenzoic acid (HBA) yield on the other hand higher $V_{\mathrm{oc}}$. There is a clear linear dependence of the obtained $V_{\text {oc }}$ with individual molecules dipole moment, suggesting that both the density of dipoles and orientation angle is similar in these SAMs. The rate of change of $V_{\mathrm{oc}}$ with dipole moment, calculated from the best linear fit, yields a value of $104 \mathrm{mV} \mathrm{D}^{-1}$. This trend is further confirmed by the $V_{\text {oc }}$-CPD shift dependence (ESI S3 $\dagger$ ). A maximum PCE of $0.11 \%$ with a $V_{\mathrm{oc}}$ of $0.56 \mathrm{~V}$ is obtained for HBA. The relevant figures of merit of this set of devices are summarized in Table S6. $\dagger$

On top of the ability of the SAMs to control the open-circuit voltage in these devices, we interestingly found that a simultaneous control can be exerted over the hot electron injection process and subsequently to $J_{\text {sc }}$. Fig. 6a depicts how hot electron efficiency varies with the selected molecule that constitute the SAM. Fig. 6b shows the IPCE peak dependence on the chemical species of the molecules in the vicinity of the metal. An evident increase from $2.6 \%$ up to $4.5 \%$ for HBA (corresponding to $0.273 \pm 0.040$ to $0.440 \pm 0.03 \mathrm{~mA} \mathrm{~cm}^{-2}$ respectively) has been witnessed revealing a trend of increasing IPCE with increasing binding energy of the exposed chemisorbed functional groups with the Ag electrode. ${ }^{47}$ We hypothesize that this could either be attributed to electrical or geometrical effects: a stronger interaction of the metal-molecule interface and a subsequent higher density of tunneling states would promote hotelectron injection compared to more weakly interacting interfaces; ${ }^{48}$ in the same way the differences at the SAM/Ag interface arising as a result of the different interaction of the exposed species with the metal during deposition could yield to different interface morphologies (i.e. metal penetration into the SAM and molecule/metal binding configuration) and thus IPCE and $J_{\mathrm{sc}} \cdot{ }^{49,50}$ This is supported by the blue-shift observed in the IPCE of MBA devices. In this configuration, the highly
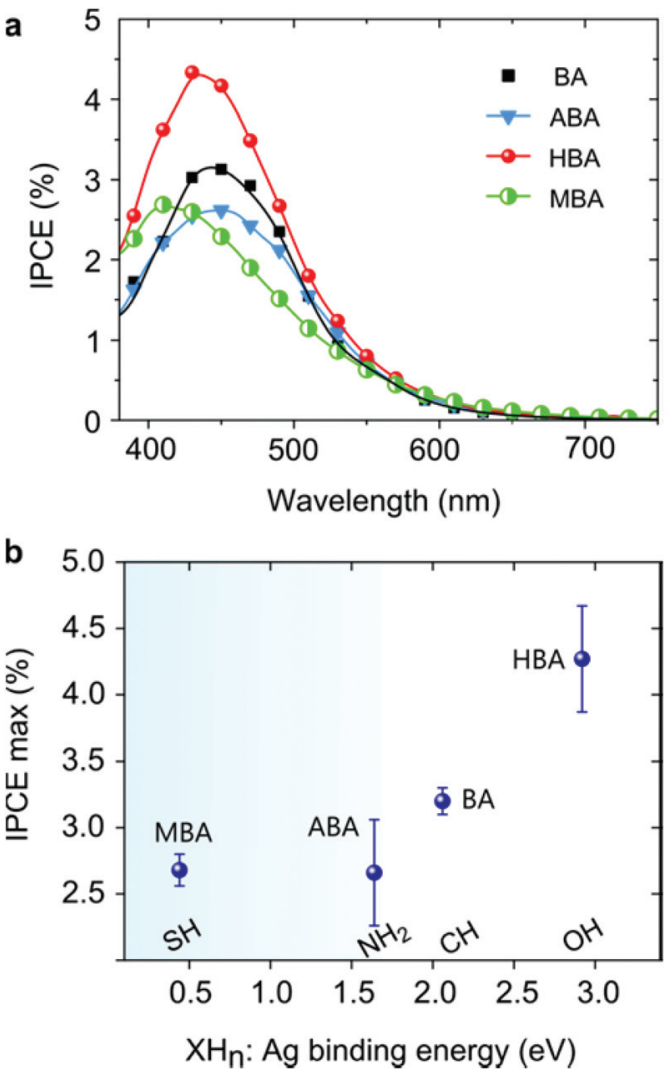

Fig. 6 Molecular dipoles to control hot-electron injection. (a) IPCE for different SAM showing and its dependence with monolayer constituent molecules. (b) The injection efficiency was found to follow a trend with the binding energy between the exposed functional group and the metal electrode.

reactive thiol group may reduce the penetration of metal atoms through the SAM during the electrode deposition, resulting in a lower refractive index environment and a subsequently blueshifted resonance elucidating the underlying mechanisms behind this new exciting functionality merits further investigation and will be subject of future work. We note that the aforementioned trends in solar cell performance are also maintained for the case of gold electrodes (see ESI S7†), thus enabling molecular interfacial control as a promising tool for hot-electron plasmonic optoelectronics.

\section{Conclusions}

In summary, we have shown that SAMs is a versatile platform to tune the metal-semiconductor interface in order to improve the photovoltaic response of plasmonic hot-electron solar cells. Molecular linkers can serve to passivate localized midgap states detrimental to the photovoltaic performance of these devices also offering the extra functionalities to tune the optoelectronic properties that go beyond the capabilities of inorganic layers. The injection efficiency can be tailored by morphological or electrochemical factors, either by adjusting the length or the functionality of the constituent molecules. By 
doing so, we report the highest $J_{\text {sc }}$ and IPCE (over 5\%) in solid state plasmonic hot-electron solar cells. We have also shown that the open-circuit voltage can be further optimized (at a rate of $\sim 100 \mathrm{mV} \mathrm{D}^{-1}$ ) by selecting a set of molecules with a proper dipole moment. This allows the simultaneous achievement of high open-circuit voltages $(0.56 \mathrm{~V})$ and fill-factors (0.58). Compared to their best inorganic passivation scheme counterparts (ref. 9), or to other plasmonic hot-electron architectures, ${ }^{19,51}$ this represents a significant improvement for plasmonic hotelectron plasmonic based photovoltaics both in terms of $J_{\mathrm{sc}}, V_{\mathrm{oc}}$ and IPCE. The use of molecular layers to modify interfacial properties opens also exciting avenues in hot-electron photodetectors, where the height of the Schottky barrier, ultimately limiting up to what extent IR light could be harvested, ${ }^{26}$ can now be tailored. Further functionalities could be envisioned by studying the interaction of the metal hot electron population with particular molecular linkers potentially enabling coherent and/or resonant transport.

\section{Experimental section}

\section{Substrates fabrication}

ITO coated glass substrates were purchased from Stuttgart University and were cleaned with acetone, ethanol and DI water under sonication 10 minutes each in sequence before use. FTO coated glass substrates were purchased from Xop Fisica and cleaned with the same procedure. $\mathrm{TiO}_{2}$ films were deposited on top of FTO substrates as reported elsewhere. Flat devices were fabricated by atomic layer deposition (ALD) of $80 \mathrm{~nm}$ of $\mathrm{TiO}_{2}$ over ITO substrates (Savannah 200, Cambridge Nanotech).

\section{SAM functionalization}

$\mathrm{TiO}_{2}$ substrates were incubated for $24 \mathrm{~h}$ in solutions of the different molecules in toluene. All substrates were annealed at $200{ }^{\circ} \mathrm{C}$ for 30 minutes prior to incubation to remove traces of moisture. The concentration was kept constant for all molecules at $0.2 \mathrm{M}$ when possible, otherwise limited by the saturation solubility of each compound. Incubation baths were stirred periodically before sample cleaning. $\mathrm{TiO}_{2}$ functionalized substrates were cleaned by vigorously rinsing them in toluene to remove ungrafted molecules. Reincubation and cleaning processes were repeated for 3 times to ensure monolayer coverage.

\section{Electrode deposition}

200-300 nm of silver were deposited on a Kurt J. Lesker Nano 36 system. The evaporation conditions were critical so as to avoid junction damage, which would lead to short-circuited or unpassivated devices. The chamber base pressure was kept lower than $1 \times 10^{-6} \mathrm{mbar}$ for all evaporations. A low evaporation rate was required for at least the first $40 \mathrm{~nm}$ (always bellow $0.3 \AA \mathrm{s}^{-1}$ ) after which it could be increased up to $1 \AA \mathrm{s}^{-1}$ for the remaining thickness. The chamber temperature was monitored and kept bellow $50^{\circ} \mathrm{C}$. At higher chamber tempera- tures device performance deteriorated which we attributed to increased metal penetration and induced defects within the SAM in view of the higher kinetic energy of metal clusters incident on the devices. The final thickness and deposition rates were controlled with a quartz crystal sensor. A shadow mask with $2 \mathrm{~mm}$ diameter circles was used to define the contact pads area.

\section{SAMs characterization}

After incubation, the presence of the SAMs was assessed by FTIR spectroscopy (Agilent FTIR spectrometer 660 attached to an optical microscope and through a 15X Cassegrain objective). Prior to that, samples were kept in a vacuum chamber for $30^{\prime}$ at a $1 \times 10^{-6}$ mbar base pressure to reproduce pre-electrode deposition conditions. After electrode deposition the vibrational signatures of the SAM were acquired with a Renishaw inVia Raman microscope. Samples were illuminated with a $532 \mathrm{~nm}$ laser excitation from the FTO side and confocally focused on the $\mathrm{TiO}_{2}-$ metal interface; the measured Raman spectrum had a range from 100 to $5000 \mathrm{~cm}^{-1}$, with a resolution of $0.6 \mathrm{~cm}^{-1}$. The integrity of the SAM after metal deposition was also corroborated with ATR-FTIR measurements over a thin, $3 \mathrm{~nm} \mathrm{Ag}$ layer. The potential presence of pinholes within the SAM could not be quantified due to the intrinsic roughness and grain size of the $\mathrm{TiO}_{2}$ substrates employed for our solar cells, which are on the order of $80 \mathrm{~nm}$. Even in the presence of defects in the SAM, the electronic properties of the interface can be dominated by the properties of the SAM provided those defects are scarce and small enough. ${ }^{52}$ The systematic correlation observed between SAM properties such as length and dipole with the figures of merit of our solar cells suggests that the presence of pinholes do not dominate or limit their performance.

\section{Device characterization}

All device characterization was performed in ambient conditions. Current-voltage characteristics were recorded using a Keithley 2400 source meter. Illumination intensity of AM 1.5 was accomplished using a solar simulator (Newport Oriel Sol3A class AAA). A shadow mask was placed just before our device so that incident light area matches with the device area. The spectral characterization was carried out by illuminating the devices with by a Newport Cornerstone 260 monochromator, modulated with a $20 \mathrm{~Hz}$ chopper, and monitoring the short circuit current with a Stanford Research System SR830.

\section{Molecule modeling and dipole moments calculation}

All molecules' coordinates were retrieved from the HIC-Up online database (Uppsala University, http://xray.bmc.uu.se/ hicup/). Their geometries were subsequently optimized with a Broyden-Fletcher-Goldfarb-Shanno algorithm and dipole moments calculated theoretically using AM1 parametrization with the semi-empirical quantum chemistry program Arguslab. ${ }^{53}$ 


\section{Acknowledgements}

We acknowledge financial support from Fundació Privada Cellex Barcelona, the European Commission's Seventh Framework Programme for Research under Contract PIRG06GA-2009-256355 and the Ministerio de Ciencia e Innovación under Contract no. TEC2011-24744. We also acknowledge support from the Nanophotonics for Energy Network of Excellence under contract N4E GA.248855. A.M was supported by an ICFONEST fellowship (COFUND program) and G.K. by a Ramon y Cajal fellowship.

\section{Notes and references}

1 J. N. Anker, W. P. Hall, O. Lyandres, N. C. Shah, J. Zhao and R. P. Van Duyne, Nat. Mater., 2008, 7, 442-453.

2 G. Konstantatos and E. H. Sargent, Nat. Nanotechnol., 2010, 5, 391-400.

3 H. A. Atwater and A. Polman, Nat. Mater., 2010, 9, 205-213.

4 D. Paz-Soldan, A. Lee, S. M. Thon, M. M. Adachi, H. Dong, P. Maraghechi, M. Yuan, A. J. Labelle, S. Hoogland, K. Liu, E. Kumacheva and E. H. Sargent, Nano Lett., 2013, 13, 1502-1508.

5 Q. Gan, F. J. Bartoli and Z. H. Kafafi, Adv. Mater., 2013, 25, 2385-2396.

6 J. M. Luther and J. L. Blackburn, Nat. Photonics, 2013, 7, 675-677.

7 F. Pelayo García de Arquer, F. J. Beck, M. Bernechea and G. Konstantatos, Appl. Phys. Lett., 2012, 100, 043101.

8 P. Senanayake, C.-H. Hung, J. Shapiro, A. Lin, B. Liang, B. S. Williams and D. L. Huffaker, Nano Lett., 2011, 11, 5279-5283.

9 F. P. García de Arquer, A. Mihi, D. Kufer and G. Konstantatos, ACS Nano, 2013, 7, 3581-3588.

10 Y. Tian and T. Tatsuma, Chem. Commun., 2004, 1810-1811.

11 M. W. Knight, H. Sobhani, P. Nordlander and N. J. Halas, Science, 2011, 332, 702-704.

12 Y. K. Lee, C. H. Jung, J. Park, H. Seo, G. A. Somorjai and J. Y. Park, Nano Lett., 2011, 11, 4251-4255.

13 S. Mubeen, G. Hernandez-Sosa, D. Moses, J. Lee and M. Moskovits, Nano Lett., 2011, 11, 5548-5552.

14 P. Reineck, G. P. Lee, D. Brick, M. Karg, P. Mulvaney and U. Bach, Adv. Mater., 2012, 24, 4750-4755.

15 F. Wang and N. A. Melosh, Nat. Commun., 2013, 4, 1711.

16 H. Chalabi, D. Schoen and M. L. Brongersma, Nano Lett., 2014, 14, 1374-1380.

17 C. Clavero, Nat. Photonics, 2014, 8, 95-103.

18 M. Aeschlimann, M. Bauer and S. Pawlik, Chem. Phys., 1996, 205, 127-141.

19 S. Mubeen, J. Lee, W.-R. Lee, N. Singh, G. D. Stucky and M. Moskovits, ACS Nano, 2014, 8, 6066-6073.

20 R. Jiang, B. Li, C. Fang and J. Wang, Adv. Mater., 2014, 26, 5274-5309.

21 T. P. White and K. R. Catchpole, Appl. Phys. Lett., 2012, 101, 073905.
22 A. J. Leenheer, P. Narang, N. S. Lewis and H. A. Atwater, J. Appl. Phys., 2014, 115, 134301.

23 G. V. Hartland, Chem. Rev., 2011, 111, 3858-3887.

24 A. O. Govorov, H. Zhang and Y. K. Gun'ko, J. Phys. Chem. C, 2013, 117, 16616-16631.

25 A. Manjavacas, J. G. Liu, V. Kulkarni and P. Nordlander, ACS Nano, 2014, 7630-7638.

26 A. Sobhani, M. W. Knight, Y. Wang, B. Zheng, N. S. King, L. V. Brown, Z. Fang, P. Nordlander and N. J. Halas, Nat. Commun., 2013, 4, 1643.

27 A. Vilan, A. Shanzer and D. Cahen, Nature, 2000, 404, 166168.

28 A. Vilan, J. Ghabboun and D. Cahen, J. Phys. Chem. B, 2003, 107, 6360-6376.

29 O. Yaffe, L. Scheres, S. R. Puniredd, N. Stein, A. Biller, R. H. Lavan, H. Shpaisman, H. Zuilhof, H. Haick, D. Cahen and A. Vilan, Nano Lett., 2009, 9, 2390-2394.

30 J. Krüger, U. Bach and M. Grätzel, Adv. Mater., 2000, 12, 447-451.

31 P. Chen, J. H. Yum, F. De Angelis, E. Mosconi, S. Fantacci, S.-J. Moon, R. H. Baker, J. Ko, M. K. Nazeeruddin and M. Grätzel, Nano Lett., 2009, 9, 2487-2492.

32 Z. He, C. Zhong, X. Huang, W.-Y. Wong, H. Wu, L. Chen, S. Su and Y. Cao, Adv. Mater., 2011, 23, 4636-4643.

33 A. Tada, Y. Geng, Q. Wei, K. Hashimoto and K. Tajima, Nat. Mater., 2011, 10, 450-455.

34 A. S. Erickson, N. K. Kedem, A. E. Haj-Yahia and D. Cahen, Appl. Phys. Lett., 2012, 101, 233901.

35 L. Barnea-Nehoshtan, P. K. Nayak, A. Shu, T. Bendikov, A. Kahn and D. Cahen, ACS Appl. Mater. Interfaces, 2014, 6, 2317-2324.

36 Q. Qu, H. Geng, R. Peng, Q. Cui, X. Gu, F. Li and M. Wang, Langmuir, 2010, 26, 9539-9546.

37 H. Zhang and A. O. Govorov, J. Phys. Chem. C, 2014, 118, $7606-7614$.

38 B. A. Gregg, F. Pichot, S. Ferrere and C. L. Fields, J. Phys. Chem. B, 2001, 105, 1422-1429.

39 M. Graetzel, R. A. J. Janssen, D. B. Mitzi and E. H. Sargent, Nature, 2012, 488, 304-312.

40 A. Vilan and D. Cahen, Adv. Funct. Mater., 2002, 12, 795807.

41 B. de Boer, M. M. Frank, Y. J. Chabal, W. Jiang, E. Garfunkel and Z. Bao, Langmuir, 2004, 20, 1539-1542.

42 H. Haick and D. Cahen, Prog. Surf. Sci., 2008, 83, 217261.

43 Y. Jin, N. Friedman, M. Sheves and D. Cahen, Langmuir, 2008, 24, 5622-5626.

44 B. de Boer, M. M. Frank, Y. J. Chabal, W. Jiang, E. Garfunkel and Z. Bao, Langmuir, 2004, 20, 1539-1542.

45 S. Rühle, M. Greenshtein, S.-G. Chen, A. Merson, H. Pizem, C. S. Sukenik, D. Cahen and A. Zaban, J. Phys. Chem. B, 2005, 109, 18907-18913.

46 C. Goh, S. R. Scully and M. D. McGehee, J. Appl. Phys., 2007, 101, 114503.

47 D. B. Kokh, R. J. Buenker and J. L. Whitten, Surf. Sci., 2006, 600, 5104-5113. 
48 A. Salomon, T. Böcking, J. J. Gooding and D. Cahen, Nano Lett., 2006, 6, 2873-2876.

49 Q. Sun, A. Selloni and G. Scoles, J. Phys. Chem. B, 2006, 110, 3493-3498.

50 F. Chen, X. Li, J. Hihath, Z. Huang and N. Tao, J. Am. Chem. Soc., 2006, 128, 15874-15881.
51 H. Lee, Y. K. Lee, E. Hwang and J. Y. Park, J. Phys. Chem. C, 2014, 118, 5650-5656.

52 H. Haick, M. Ambrico, T. Ligonzo, R. T. Tung and D. Cahen, J. Am. Chem. Soc., 2006, 128, 6854-6869.

53 ArgusLab - http://www.arguslab.com. 